\title{
BMJ Global Health Associations between bride price obligations and women's anger, symptoms of mental distress, poverty, spouse and family conflict and preoccupations with injustice in conflict-affected Timor-Leste
}

To cite: Rees S, Mohsin M, Tay AK, et al. Associations between bride price obligations and women's anger, symptoms of mental distress, poverty, spouse and family conflict and preoccupations with injustice in conflict-affected TimorLeste. BMJ Global Health 2016:1:e000025

doi:10.1136/bmjgh-2015000025

Received 15 December 2015 Revised 30 March 2016 Accepted 24 April 2016

\section{CrossMark}

${ }^{1}$ Psychiatry Research and Teaching Unit, University of New South Wales and Academic Mental Health Unit, South Western Sydney Local Health District, Liverpool Hospital, Sydney, New South Wales, Australia

2James Cook University, Townsville, Queensland, Australia

${ }^{3}$ Alola Women's Foundation, Dili, Timor-Leste

${ }^{4}$ Bloomberg School of Public Health, Johns Hopkins University, Baltimore,

Maryland, USA

Correspondence to

Dr Susan Rees;

s.j.rees@unsw.edu.au

\section{ABSTRACT}

Objectives: Bride price is a widespread custom in many parts of the world, including in most countries in sub-Saharan Africa and parts of Asia. We hypothesised that problems relating to the obligatory ongoing remittances made by the husband and his family to the bride's family may be a source of mental disturbance (in the form of explosive anger and severe mental distress) among women. In addition, we postulated that problems arising with bride price would be associated with conflict with the spouse and family, poverty and women's preoccupations with injustice. Design: A mixed-methods study comprising a total community household survey and semistructured qualitative interviews.

Setting: Two villages, one urban, the other rural, in Timor-Leste.

Participants: 1193 married women participated in the household survey and a structured subsample of 77 women participated in qualitative interviews.

Results: Problems with bride price showed a consistent dose-effect relationship with sudden episodes of explosive anger, excessive anger and severe psychological distress. Women with the most severe problems with bride price had twice the poverty scores as those with no problems with the custom. Women with the most severe problems with bride price also reported a threefold increase in conflict with their spouse and a fivefold increase in conflict with family. They also reported heightened preoccupations with injustice.

Conclusions: Our study is the first to show consistent associations between problems with bride price obligations and mental distress, poverty, conflict with spouse and family and preoccupations with injustice among women in a low-income, postconflict country.

\section{BACKGROUND}

The promotion of gender equality represented one of the eight global Millennium

\section{Key questions}

What is already known about this topic?

- There is an ongoing debate about the risk that bride price poses to the general and menta health of women in developing countries.

- In Timor-Leste, bride price (incorporated into the traditional practice of barlake) includes an obligation that the husband and his family have to provide money or material goods to the wife's family, including for life events such as future weddings and funerals.

- This form of bride price commonly involves a long-term obligation which requires payment over extended periods of time.

What are the new findings?

- Problems with bride price were strongly associated with sudden episodes of explosive anger, experiencing excessive anger and severe psychological distress among women.

- Women with the most severe problems with bride price reported twice the level of poverty as those with no problems with bride price.

- There was a threefold increase in conflic with the male spouse and a fivefold increase in conflict within the family among women with the most severe problems with bride price.

Recommendations for policy

- Bride price should be considered as a risk factor for mental health problems among women in low-income countries.

- Bride price should be considered in all family interventions, particularly where there is family conflict.

- Training of mental health professionals and those working in psychosocial support services should include an understanding of the impact of bride price on mental health. 
Development Goals (MDGs), the overall objective being to advance the status of women and promote their participation in socioeconomic development in low-income and middle-income countries. The importance of promoting gender equality and the empowerment of women has been further underlined by the inclusion of these objectives in the recently adopted Sustainable Development Goals. ${ }^{1}$ Achieving these goals has remained elusive, however, particularly in low-income, conflict-affected countries. ${ }^{2}$ One obstacle may be the ongoing problems families and women in particular encounter in meeting traditional obligations, such as the ceremonial bride price. We report a mixed-methods study in Timor-Leste investigating whether problems with bride price were associated with poor mental health (symptoms of explosive anger, experiencing excessive anger and general mental distress), family problems (conflict with spouse and family, poverty) and heightened preoccupations with injustice among women.

\section{Bride price}

There is an ongoing debate, particularly in Africa and Southeast Asia, about the risks that the practice of bride price pose to the security, health and status of women. ${ }^{3}$ Although the bride price custom may have positive effects, for example, by encouraging parental investment in their daughter's education, ${ }^{4}$ critics point out that the practice tends to 'commodify' women by representing them as objects of transaction, thereby increasing risk of early marriage, subsequent reproductive health problems (including vulnerability to HIV-AIDS) and ill treatment by the indebted husband and/or his family. ${ }^{4-8}$

Bride price is often inter-related with a complex system of material remittances in traditional societies. In Timor-Leste, the term barlake encompasses more than a bride price in that it refers to a range of ritual contributions as well as exchanges made between the fetosan (husband's family) and the umane (wife's family). ${ }^{9}$ In the prevailing patriarchal system, the bride's family or clan is commonly categorised as wife-givers (or lifegivers, manegsan/umane) and the husband's family as wife-takers (or life-takers, fetosan). This complex practice of bride price obliges the husband's family to offer the wife's family gifts, offerings and contributions of traditional items such as livestock, food, handmade craft and jewellery for ceremonies and other significant life events such as marriage and funerals, throughout the period of marriage. ${ }^{9}$ In addition, at the time of the marriage, gifts are exchanged, although the most valuable are generally provided by the groom. ${ }^{10}$ Barlake (also known as lia) commonly involves remittances made over extended periods of time from the husband's family to the bride's family, and in the modern context, often involves cash payments.

Barlake has the positive effect of acting as a symbol of mutual respect between families brought together by marriage, providing material assistance to family members in need of support, and establishing clarity regarding future inheritance rights. The responsibility of meeting obligations also has the function of committing the fetosan (husband) to valuing and ensuring the safety and well-being of the new wife. ${ }^{9}{ }^{11}$ Nevertheless, it is possible that the protracted nature of the husband's obligations to offer gifts or outlays to the wife and her family, and the growing cost of this form of bride price in the modern cash economy, may have adverse consequences over time, including increasing resentment within the wider network ${ }^{9}{ }^{11}$ and potentially conflict with and even violence against the female spouse. ${ }^{10}$

Owing to the sociocultural complexities and regional variations in the use of the terms barlake and lia in Timor-Leste, for the purposes of our study, we operationalised the terms used in our inquiry to represent the core construct of bride price. While most often referred to by participants in the region of our study as lia, we ensured that the definition we used was clearly understood to mean the husband's and/or his family's obligations to make material contributions (money or other goods and items) to the bride's family for cultural, social or ritual purposes, including commitments that might extend over a prolonged period of time following the marriage. We provide further information about our operationalisation of bride price in the Method section.

\section{Poverty and bride price}

The prolonged period of war and conflict in Timor-Leste has left many families in a state of extreme poverty, with Timor-Leste ranked as one of the poorest nations in the world. ${ }^{9}$ Obligations related to bride price therefore may exacerbate already strained family resources, impacting on the capacity of the woman to meet basic necessities including food and the educational, housing and healthcare needs of the family. ${ }^{11}{ }^{12}$ As with many cultural practices, the mode of discharging bride price has evolved over time, being subject to the effects of rapid social and economic change typical of postconflict countries such as Timor-Leste. Traditionally, bride price was paid in domestic animals, for example, in cattle in Zambia, and water buffalo in Timor-Leste. $^{11}{ }^{13}$ Nevertheless, social and economic development and the shift to a cash economy have meant that in contemporary times, bride price is commonly paid in monetary currency, a transition associated with a rapid inflation in the cost to the husband and his family. The contemporary practice of bride price is therefore attended by several risks: financial pressures, particularly in conditions of poverty in low-income, conflict-affected countries such as Timor-Leste; an incentive for parents to 'sell' their daughters prematurely; the potential for recipients to demand that bride price debts are discharged more rapidly to meet urgent debts or immediate needs; ${ }^{14}$ entrapping women in a violent marriage for fear of parents having to repay the large bride price; and in modernising environments, generating competing demands within families, for example, to fund their children's ongoing education as 
opposed to saving for a future bride price. The prolonged period of obligation associated with bride price in Timor-Leste, in particular, may lead to mounting resentment by the indebted husband and his relatives, increasing the risk of conflict among spouses and the wider family. ${ }^{5} 71115$

\section{Bride price and mental health}

Little attention has been given in the research literature to the relationship between bride price obligations and women's mental health in low-income and mediumincome countries. The only relevant data we could identify were gathered in a cross-country study whose primary focus was on domestic violence. A subsidiary finding from that inquiry was that dowry obligations showed no association with suicide attempts among women. ${ }^{16}{ }^{17}$ It is possible, however, that problems associated with bride price contribute to more general and common forms of mental distress among women, as suggested by preliminary data we have gathered in Timor-Leste. ${ }^{18}$ Background data from our programme of research in Timor-Leste has documented high rates of explosive anger among men and women in that country, a reaction pattern that is associated with a range of general factors, including past exposure to traumas related to human rights violations, ongoing deprivations and feelings of injustice. ${ }^{15}$ In our qualitative research, however, women specifically highlighted the problem of bride price as a contributor to poverty, a source of conflict with the spouse and family and a possible cause of their anger. ${ }^{18}$ These preliminary findings warranted a closer examination of the possible role of bride price as a factor leading to mental distress among Timorese women.

\section{Setting of conflict and poverty}

Timor-Leste was invaded and occupied by Indonesia in 1975, provoking a protracted period of conflict and persecution that culminated in the humanitarian emergency of 1999. During the period of conflict, it is estimated that a quarter of the indigenous population (of 600000 persons) died as a consequence of atrocities, warfare, burning of villages, murder, famine and untreated illness. ${ }^{19}$ In addition, there was widespread loss of property and livelihoods, and extensive forced displacement of whole communities. In the postconflict period, further episodes of internal violence, particularly in 2006-2007, led to injuries, deaths and internal displacement of communities. Socioeconomic development in the newly independent country has been slow, with many families confronting extreme levels of poverty and deprivation.

The present inquiry forms part of a programme of mental health studies that our team has undertaken in Timor-Leste over two decades. The quantitative data for the present analysis were drawn from a second survey of two villages conducted in 2010-2011, undertaken concurrently with a qualitative study whose aim was to understand more fully the manifestations and sources of women's anger. ${ }^{15}{ }^{18}$ In the present analysis, we hypothesised that problems Timorese women experienced with bride price would be associated with explosive anger (the prevalence and excessive nature of the reaction) as well as with more general symptoms of severe psychological distress. We also postulated that problems with bride price would be associated with several adverse psychosocial consequences relevant to women's mental health, including conflict with the spouse and family, ongoing poverty and distressing preoccupations with injustice.

\section{METHOD}

The mixed methods approach included an epidemiological survey and a qualitative component. Qualitative and quantitative interviews were completed on separate days with different interviewers employed for each component. Qualitative interviewers were not aware of trends or findings from the quantitative study until after its completion. In our Quant-QUAL analysis, ${ }^{20}$ we first examined for quantitative associations between bride price and indices of explosive anger and mental distress, and then with relevant psychosocial factors including poverty, preoccupations with injustice, conflict with family and conflict with spouse. ${ }^{15} 18$ Our focus was on whether there was a regular dose-effect relationship between problems with bride price and all our indices of mental distress and psychosocial difficulties. The subsequent qualitative analysis aimed to explore further the contextual nature and sequencing of these factors from the perspective of women, specifically examining how bride price intersected with other factors (poverty, preoccupation with injustice related to past conflict and contemporary hardship, and conflict with spouse and family) in generating distress.

\section{The epidemiological sample}

All adults, 18 years or older, living in all dwellings in each of two administrative units or sucos were eligible for inclusion. The sucos included an urban settlement in Dili, the capital, and a rural site situated $\sim 1$ hour's drive from the urban area. Global positioning system (GPS) coordinates and maps generated by the National Directorate of Statistics allowed us to identify all dwellings in the two villages. Excluded were those who were too ill or incapacitated to participate including those with overt psychosis, intellectual impairment or organic brain disorder such as severe dementia. We achieved a $92.6 \%$ response from the eligible women approached. Given that the focus of the study was on the personal experiences of bride price among women, we excluded women who were single or never married $(n=320)$ from the whole sample $(n=1513)^{15}$ for the present analysis, yielding a final analytic sample of 1193 women. 
Personnel and training for the epidemiological survey

The quantitative survey was undertaken by 18 Timorese fieldworkers with prior survey experience and/or public health degrees, supervised by expatriate staff. All field staff received 2 weeks' research training followed by 2 months of field testing and questionnaire piloting in areas separate from the sites of the main study. Pairs of interviewers were required to achieve a consistent $100 \%$ level of inter-rater reliability with supervisors on key mental health measures. The survey was conducted between June 2010 and October 2011.

\section{The qualitative sample}

We assessed the data available from the epidemiological study to identify a sample of women $(n=77)$ weighted towards those meeting full criteria for explosive anger $(\mathrm{n}=64)^{18}$ (defined according to the criteria of intermittent explosive disorder (IED), as outlined hereunder). The inclusion of a minority of women without IED allowed us to gather observer perspectives from participants not personally affected by explosive anger or aggression. The qualitative component was undertaken in accordance with the Consolidated Criteria for Reporting Qualitative Research (COREQ) checklist, ${ }^{21}$ the approach aiming to facilitate a safe exploration of gender-sensitive issues in a setting where cultural and religious factors might constrain disclosure of personal information. The full methodology has been reported previously. ${ }^{18}$

\section{Personnel and procedure for the qualitative study}

For the qualitative research, an Australian project director and a Timorese in-country manager supervised three fieldworkers who were trained in maternal and child health at the Alola Foundation, the peak nongovernment agency for women in Timor-Leste. In conducting the qualitative interviews, we applied strict provisions of confidentiality and privacy according to WHO guiding principles. ${ }^{22}$ In brief, phase 1 of the qualitative study involved open-ended and in-depth interviews with 19 of the 77 women exploring domains informed by the existing literature (non-peer-reviewed reports and peerreviewed publications) pertaining to the psychosocial conditions of women in Timor-Leste, explosive anger and the manifestations and factors associated with that emotion. Phase 2 involved 58 participants and included a semistructured interview designed to confirm the factors associated with anger that were compiled from the data gathered in phase 1 , and to establish more fully how and why these factors might precipitate or aggravate women's anger. This included a further examination of bride price given that this issue was raised repeatedly by women in the phase 1 interviews. Phase 3 involved a professional sample focus group with workers from government and non-government organisations in Timor-Leste working specifically with women, including in the violence against women and mental health sectors. Data generated from the field interviews were discussed, clarified and checked by way of feedback from this professional sample.

Measures for the quantitative survey

\section{Bride price}

As indicated, we derived our definition of bride price from preliminary qualitative work identifying culturally specific practices of barlake and lia. ${ }^{18}$ To ensure precision, we operationalised bride price for the purposes of the study as the customary practice in which the husband and his family were obliged to make remittances of money or other goods to the bride's family for cultural, social or ritual purposes, a commitment that often extended over prolonged periods of time following the marriage.

\section{Explosive anger}

The community measure of explosive anger, based on the Diagnostic and Statistical Manual edition IV (DSM-IV) criteria for IED, was tested and modified serially during piloting to ensure its cultural appropriateness and comprehensibility in the local language, Tetum. ${ }^{18}$ The measure identified sudden attacks of explosive anger and associated acts of aggression including loss of control, verbal aggression, destruction of property and physical aggression towards others. The measure was assessed in a convergence study against IED determined by the Structured Clinical Interview for DSM-IV applied in a blinded manner by experienced expatriate psychologists familiar with Timor-Leste, working with interpreters. ${ }^{22}$ There was a high level of concordance between the two measures for assigning IED: area under the curve (AUC) 0.90 (95\% CI 0.83 to 0.98). ${ }^{22}$ In the present study, we focused on two indices, sudden attacks of explosive anger $(0=$ absence, $1=$ presence) and attacks judged to be excessive by the women $(0=$ attacks not excessive; $1=$ attacks experienced as excessive).

\section{Severe psychological distress}

We included the Kessler-10 scale $(\mathrm{K} 10)^{23}$ as a measure of severe psychological distress, the index comprising symptoms of depression, anxiety and somatic complaints. Past studies have indicated that the K10 provides a general index of mental disorder. ${ }^{23} 24$ Our aforementioned study based on a stratified sample of 97 community respondents drawn from our household survey in Timor-Leste compared the K10 with the category of major depressive disorder of the Structured Clinical Interview for DSM-IV, applied in a blinded manner by experienced psychologists. ${ }^{22}$ There was a sound level of convergence between the K10 and the gold standard interview: AUC for the K10 0.79 (95\% CI 0.67 to 0.91 ) (full data will be provided on request). The international cut-off score of 30 or more provided the best balance between sensitivity $(92.3 \%)$ and specificity $(66 \%)$. The lower level of specificity was most likely related to the structure of the K10 which includes 
symptoms of anxiety and somatic symptoms in addition to depression.

\section{Daily living difficulties}

A list of 27 items was included in the household survey to measure living difficulties that had been experienced over the past 12 months. Items were identified following extensive community consultation. A subset of seven items was used to generate a poverty index. Poverty items included: not enough food; not enough money in general; not enough money for school fees; having to work too hard; poor shelter; unemployment; and being too old to work. Each item was scored on a scale of 1-5: (1) not a problem, (2) a bit of a problem, (3) a moderately serious problem, (4) a serious problem and (5) a very serious problem. The total poverty score reflected the sum of all item scores.

\section{Conflict with spouse and conflict with family}

Conflict with spouse and conflict with family were individually measured by single-item questions asking if each occurred and if so, whether they were a moderate, serious or a very serious problem.

\section{Preoccupations with injustice}

To assess preoccupations with injustice, we asked respondents to identify and describe the worst acts or conditions of injustice they had experienced in each of the three defined historical periods: the Indonesian occupation, the period of internal conflict and in contemporary times. In most instances, qualitative data indicated that the focus of respondents' preoccupations with injustice related to past human rights violations and, in relation to contemporary times, ongoing deprivations. The composite measure covering three successive historical periods therefore reflects a general index of the preoccupation with injustice the person experienced, feelings which, we hypothesised, could be exacerbated by grievances related to bride price obligations. Responses were scored as 0 for no event nominated; 1 for the experience of an unjust event; 2 for experience of an event and persisting preoccupations of injustice; and 3 for an event experienced, persisting preoccupations of injustice and associated ongoing distress related to the preoccupations. In our analysis, we included a composite index of injustice based on the summary respondent scores (0-3) for all three historical periods.

\section{Ethics and partnerships}

The study was undertaken in partnership with the Alola Foundation. The Human Research Ethics Committee of the University of New South Wales and the Timor-Leste Ministry of Health approved the study. The information sheet outlined the objectives and was read to all participants because of low literacy in the community. For the same reason, we requested verbal consent. Participants then made the choice to provide verbal informed consent in the presence of a respected Timorese person and the interview staff. The interviewer signed the consent form stating that the participant had understood the information sheet and had agreed voluntarily to participate. The process for verbal consent to participate was approved by The Human Research Ethics Committee of the University of New South Wales and the Timor-Leste Ministry of Health. The study was funded by grants from the Australian National Health and Medical Research Council (Grant 568732) and the Australian Research Council (DP0987803).

\section{Statistical analyses}

We present descriptive statistics for sociodemographic characteristics and mental health and psychosocial indices. We apply bivariate analyses to examine associations between problems with bride price and indices of mental health including explosive anger (two indices, one based on sudden explosive anger attacks, the second on the excessiveness of attacks), and threshold for severe psychological distress $(\geq 30)$. Bivariate analyses were also used to explore associations between problems with bride price and conflict with a spouse, conflict with family, preoccupations with injustice and ongoing poverty. The results of the bivariate analyses were expressed as percentages, $\chi^{2}$ tests and $\mathrm{F}$ tests. Univariate logistic regression was used to generate ORs with $95 \%$ CI.

For the qualitative data, interviews were independently assessed by two raters. After each rater coded the raw data, minor differences were reconciled by consultation. We used QSR NVivo $9^{25}$ for data management and analysis in order to examine dominant and dissonant themes and their inter-relationships. ${ }^{26}$ Triangulation of the key sources of data was accomplished by using the quantitative data as the scaffolding on which the qualitative data were applied to build a more comprehensive picture, the findings being crosschecked with nongovernment organisation personnel in two focus groups held following the survey, once the preliminary qualitative data had been analysed. ${ }^{26}$ The focus groups were conducted with the Alola Foundation and included representatives from health and mental health nongovernmental organisations, local women's organisations, the Ministry of Health and the Ministry for Social Solidarity. Qualitative themes and associated findings are represented in quotations. We have not included the ages of the women quoted to protect their identities. All quotations are from different participants.

\section{RESULTS}

\section{Quantitative sample characteristics}

Table 1 presents descriptive data for the quantitative survey including the sample of 1193 women. Approximately 1 in $10(11.3 \%)$ of the sample was 25 years or younger, a third $(36 \%)$ was aged $25-34$ years and $23 \%$ were 50 years or older (table 1 ). The majority $(69.6 \%)$ lived in the rural area, and $87.3 \%$ were 
Table 1 Sociodemographic and mental health characteristics of women

\begin{tabular}{|c|c|c|}
\hline Sociodemographic and mental health characteristics & Number of respondents & $\%$ of total respondents \\
\hline All & 1193 & 100.0 \\
\hline \multicolumn{3}{|l|}{ Age groups (in years) } \\
\hline $18-24$ & 135 & 11.3 \\
\hline $25-34$ & 429 & 36.0 \\
\hline $35-49$ & 354 & 29.7 \\
\hline $50+$ & 275 & 23.0 \\
\hline Mean (SD) & $39.1(14.4)$ & \\
\hline \multicolumn{3}{|l|}{ Residence } \\
\hline Rural & 830 & 69.6 \\
\hline Urban & 363 & 30.4 \\
\hline \multicolumn{3}{|l|}{ Marital status } \\
\hline Married & 1042 & 87.3 \\
\hline Divorced/separated/widowed & 151 & 12.7 \\
\hline \multicolumn{3}{|l|}{ Employment category } \\
\hline Employed with paid salary & 284 & 23.8 \\
\hline Farming/home duties/other & 859 & 72.0 \\
\hline Unemployed & 50 & 4.2 \\
\hline \multicolumn{3}{|l|}{ Level of education } \\
\hline None or primary completed & 767 & 64.3 \\
\hline Completed junior high school & 107 & 9.0 \\
\hline Completed senior high school & 262 & 22.0 \\
\hline Completed university or technical college & 57 & 4.7 \\
\hline \multicolumn{3}{|l|}{ Conflict with spouse } \\
\hline Not a problem & 633 & 53.0 \\
\hline A bit of a problem & 335 & 28.1 \\
\hline Moderately serious problem & 181 & 15.2 \\
\hline A serious/very serious problem & 44 & 3.7 \\
\hline \multicolumn{3}{|l|}{ Conflict with family } \\
\hline Not a problem & 762 & 63.9 \\
\hline A bit of a problem & 264 & 22.1 \\
\hline Moderately serious problem & 140 & 11.7 \\
\hline A serious/very serious problem & 27 & 2.3 \\
\hline \multicolumn{3}{|l|}{ Problem of bride price (lia) } \\
\hline Not a problem & 152 & 12.7 \\
\hline A bit of a problem & 442 & 37.1 \\
\hline Moderately serious problem & 368 & 30.8 \\
\hline A serious/very serious problem & 231 & 19.4 \\
\hline Sudden episodes of anger attacks & 460 & 38.6 \\
\hline Excessive anger attacks & 573 & 48.0 \\
\hline Severe psychological distress $(\mathrm{K} 10 \geq 30)$ & 189 & 15.8 \\
\hline Preoccupations with injustice ${ }^{*}$ & 857 & 71.8 \\
\hline Poverty score $†$ mean (SD) & $16.3(4.8)$ & \\
\hline
\end{tabular}

*Preoccupations with injustice represents those who experienced injustice at any time of the three following predefined periods: Indonesian times, around independence and around the time of survey (2010-2011).

†Poverty included the following seven items (each item scored 1-5: (1) not a problem, (2) a bit of a problem, (3) moderately serious problem, (4) a serious problem and (5) a very serious problem; total score ranges from 7 to 35): not enough food; not enough money; not enough money for school fees; work too hard; poor shelter; unemployment; and too old to work.

K10, Kessler-10 scale. 
married. Two-thirds $(64.3 \%)$ had either never attended school or had completed primary school only, 22\% had completed senior high school and $4.7 \%$ had obtained a degree or other postschool qualification. Fewer than a quarter $(23.8 \%)$ of women were in paid employment.

A minority $(12.7 \%)$ reported no problem with bride price, $30.8 \%$ experienced a moderately serious problem with the custom and $19.4 \%$ reported that the practice was a serious problem. Over a third $(38.6 \%)$ had sudden attacks of explosive anger and close to a half $(48 \%)$ reported attacks that they considered to be excessive. Approximately one in six (15.8\%) participants reached the threshold for severe psychological distress on the K10 (score $\geq 30$ ). One in five women $(18.9 \%$ ) reported moderate to very serious conflict with the spouse and $14.0 \%$ a comparable level of conflict with the family. A total of 7 out of 10 women $(71.8 \%)$ were preoccupied with injustice. The mean poverty score was 16.3 (range 7-35).

\section{Qualitative sample characteristics}

Of the 77 women interviewed in the qualitative study, the age range was 25-54 years, the majority $(62.3 \%)$ lived in the rural area and most $(68.9 \%)$ were married, the remainder being divorced, separated or widowed. The number of children per household ranged from 1 to 10 . Half (48\%) were occupied with subsistence farming supplemented by tending a small shop or stall selling produce.

\section{Associations between bride price and mental health measures}

The analysis reported in table 2 indicates a regular dose-effect relationship between increasing problems with bride price and all three indices of mental health problems, including sudden attacks of explosive anger, experiencing excessive anger and severe psychological distress $($ all $\mathrm{p}<0.001)$. Sudden attacks of explosive anger were almost three times as common among women with the most severe problems with bride price (55.0\%, $\mathrm{n}=127)$ compared with women with no problems with bride price $(19.7 \%, \mathrm{n}=30) \quad(\mathrm{OR}=3.395 \%$ CI 2.2 to 5.1$)$.
For severe psychological distress, there was a threefold greater prevalence for those with the most severe problems with bride price $(29.9 \%, \mathrm{n}=69)$ compared with women with no problems with the custom $(10.5 \%$, $\mathrm{n}=16)(\mathrm{OR}=3.6,95 \%$ CI 2.0 to 6.5$)$.

A participant articulated the connections she recognised between bride price (identified as lia), explosive anger and mental distress:

For me, if I feel angry I just keep quiet and go to sleep, but what makes me feel very sad is my family always come for lia. They don't care whether we have money or not. All they know is that if we get married that means that we have money, and are ready to participate for bride price at any time. We can't do anything in response because this is our tradition. Now we lost our house because of floods in January this year. We are now staying in a government-provided house and I worry because they will force us to leave this house one day.

\section{Bride price, poverty and explosive anger}

A regular dose-effect relationship was evident in examining problems with bride price and poverty $(\mathrm{F}=115.2$, $\mathrm{p}<0.001$ ) (table 3). Women with the most severe problems with bride price had twice the level of poverty (mean=25.2; 95\% CI 23.4 to 26.9) as those with no problem with the custom (mean=12.6; 95\% CI 12.0 to 13.2).

Women often used the term hanoin barak (thinking too much) to refer to intense worry or preoccupation with the problem of poverty associated with bride price.

I'm thinking a lot when I couldn't support money for my children at the time they need for their school needs and for their future and sometimes no money for lia. My relatives said that they will not help me if one day I face the same difficulty like what they have now. These things made us very angry.

A woman highlighted the interconnection of bride price, living hardships, family challenges and anger:

Women mostly get angry just because of dealing with children at home, no money, not enough food, children

Table 2 Association between problems with bride price and mental health

\begin{tabular}{|c|c|c|c|c|}
\hline Problems with bride price & $\begin{array}{l}\text { Number of } \\
\text { respondents }\end{array}$ & $\begin{array}{l}\text { Sudden attacks } \\
\text { of anger } \\
\text { Yes } \\
\text { Row \% (n) }\end{array}$ & $\begin{array}{l}\text { Anger attacks experienced } \\
\text { as excessive } \\
\text { Yes } \\
\text { Row } \%(n)\end{array}$ & $\begin{array}{l}\text { Severe psychological } \\
\text { distress } \\
\text { K10 } \geq 30 \text { (Yes) } \\
\text { Row \% (n) }\end{array}$ \\
\hline Not a problem & 152 & $19.7(30)$ & $32.950)$ & $10.5(16)$ \\
\hline A bit of a problem & 442 & $34.4(152)$ & $37.6(166)$ & $10.2(45)$ \\
\hline Moderately serious problem & 368 & $41.0(151)$ & $58.2(214)$ & $16.0(59)$ \\
\hline A serious/very serious problem & 231 & $55.0(127)$ & $61.9(143)$ & $29.9(69)$ \\
\hline Total & 1193 & $38.6(460)$ & $48.0(573)$ & $15.8(189)$ \\
\hline$p$ Values for $\chi^{2}$ tests & & $<0.001$ & $<0.001$ & $<0.001$ \\
\hline
\end{tabular}

K10, Kessler-10 scale. 
Table 3 Associations between problems with bride price and conflict with spouse, family conflict, preoccupation with injustice and poverty

\begin{tabular}{|c|c|c|c|c|c|}
\hline $\begin{array}{l}\text { Problems with } \\
\text { bride price }\end{array}$ & $\begin{array}{l}\text { Total number of } \\
\text { respondents }\end{array}$ & $\begin{array}{l}\text { Conflict with spouse } \\
\text { Moderate/serious/ } \\
\text { very serious } \\
\text { problem } \\
\text { Row \% (n) }\end{array}$ & $\begin{array}{l}\text { Conflict with family } \\
\text { Moderate/serious/ } \\
\text { very serious } \\
\text { problem } \\
\text { Row \% (n) }\end{array}$ & $\begin{array}{l}\text { Preoccupations } \\
\text { with injustice } \\
\text { Yes } \\
\text { Row \% (n) }\end{array}$ & $\begin{array}{l}\text { Ongoing poverty } \\
\text { Mean score (SD) }\end{array}$ \\
\hline Not a problem & 152 & $9.9(15)$ & $4.6(7)$ & $61.2(93)$ & $12.6(3.9)$ \\
\hline A bit of a problem & 442 & $10.4(46)$ & 7.9 (35) & $70.6(312)$ & $15.1(3.9)$ \\
\hline $\begin{array}{l}\text { Moderately serious } \\
\text { problem }\end{array}$ & 368 & $25.0(92)$ & $20.4(75)$ & 75.8 (279) & $16.8(4.0)$ \\
\hline $\begin{array}{l}\text { A serious/very } \\
\text { serious problem }\end{array}$ & 231 & $31.2(72)$ & $21.6(50)$ & 74.9 (173) & $20.1(5.1)$ \\
\hline Total & 1193 & $18.9(225)$ & $14.0(167)$ & $71.8(857)$ & $16.3(4.8)$ \\
\hline $\mathrm{p}$ Values for $\chi^{2}$ test & & $<0.001$ & $<0.001$ & $<0.001$ & $F=115.2 ; p<0.001$ \\
\hline
\end{tabular}

naughty, family demands for lia, so in the end they are violence towards the children or have problems with their husband. I used to argue with my husband just because of lia.

\section{Bride price and preoccupations with injustice}

Table 3 indicates a dose-response relationship between the severity of problems with bride price and preoccupations with injustice $(\mathrm{p}<0.001)$; those experiencing the most severe problems with bride price $(74.9 \%, \mathrm{n}=173)$ had a significant increase in preoccupations with injustice compared with those with no problems with bride price $(\mathrm{OR}=1.9,95 \%$ CI 1.2 to 2.9$)$.

The qualitative data shed light on these findings, in particular, highlighting associations of preoccupations with injustice related to war-related conflict, explosive anger and the burden of paying bride price. This quote also suggests that the payment of lia is sometimes bidirectional and related to the exchange of gifts, with the husband's family in this case wanting some of the lia in addition to the wife's family.

After the 2006 violence (the internal conflict) we fled to W. When we were there, my husband's family wanted some of our money; they all want to share lia. This made me really angry and I was having regular attacks of extreme anger and being violent.

\section{Spousal conflict, family conflict and wider implications}

A dose-effect relationship again was evident when examining associations between the severity of problems with bride price and conflict with the husband $(\mathrm{OR}=5.7$, $95 \%$ CI 2.5 to 13.0$)$ and with the family ( $\mathrm{OR}=4.1,95 \%$ CI 2.3 to 7.5 ) (both associations, p<0.01) (table 3). Specifically, rates of conflict with the male spouse increased from $9.9 \%$ for those who had no problems with bride price to $31.2 \%$ among those with the most serious problems with bride price, a threefold increase. In relation to conflict in the family, there was a fivefold increase $(4.6-21.6 \%)$ in the dose-effect relationship between the lowest and highest category of problems with bride price (table 3 ).

Qualitative data endorsed the quantitative findings on the impact of bride price on conflict with the spouse and the family, particularly in the context of poverty.

Life is so hard but sometimes we have to contribute to lia, family members always force us to bring lots of money. It created problems between my husband and me, just because of no money.

About lia my husband always complains. Every time we have lia my family always forces us (my husband and me) to give them goat and buffalo. This demand makes my husband and I always argue and angry with each other.

Those to whom bride price was owed indicated that there was a reciprocally negative effect of the practice:

I can't work because I'm too old. There is no money to participate in lia. Now my son and daughter look after me and there is no money for lia. I feel angry with them because they can't afford lia. This is our own tradition, we should do it.

A younger participant indicated the competing demands created by bride price, especially in relation to caring for children:

I don't like lia because it forces us to give a lot of money but normally I only give some because I have to first spend money on meeting the children's needs. I respect that this is our tradition, but we have to minimise it.

The tension between modern world demands and cultural traditions added pressure on the family as indicated by this quote:

Lia from my husband's family is very hard but now he is studying so they understand this condition but this is our tradition so it is impossible for us to avoid it all of the time. 
Resentment arose in trying to meet the demands imposed by bride price and dealing with contemporary family needs such as the costs associated with children's schooling:

I think we all know that in Timor when a person dies all the family from both sides only think about tradition but they don't think about the children's future.

Lia led to debt, forcing some families to borrow in order to meet their traditional obligations:

In the village, our relatives force us to contribute to lia. In response to this demand we have to borrow money to give to them... even if it is very hard, but this is our tradition.

\section{DISCUSSION}

In spite of the widespread nature of the tradition of bride price in low-income and medium-income countries, ours is the first study to explicitly examine the association of the practice with women's mental health and the broader psychosocial factors that are known to be associated with distress among that gender group. ${ }^{15}{ }^{18} \mathrm{~A}$ focus on this issue is important given that safeguarding and promoting the mental health of women are essential to advancing gender equity and particularly the capacity of women to engage fully in development in low-income and medium-income countries that are affected by conflict. ${ }^{27}$ Notably, there was a two-and-a-half-fold increase in sudden attacks of explosive anger, a twofold increase in excessive anger and a threefold increase in severe psychological distress when comparing women with the most severe problem levels with bride price with those with no problems with the practice. In those with the most severe problems with bride price, half of participating women had sudden attacks of explosive anger, three-fifths had excessive anger and nearly a third had threshold levels of severe psychological distress.

As hypothesised, there was a strong association between problems with bride price and conflict with spouse and family. Compared with those with no problems with bride price, those with the most severe problems with the practice had a threefold increase in conflict with spouse and a fivefold increase in conflict with family. In addition, the mean poverty score was twice as high for women with severe as opposed to no problems with bride price. Women with the most severe problems with bride price had twice the level of poverty (mean=25.2 vs 12.6). Of interest too was that women with the most severe problems with bride price were more preoccupied with feelings of injustice, a factor known to be associated with explosive anger. ${ }^{28}$

The strengths of the quantitative study are the large sample size, the systematic approach to recruitment and the high response rate. Women participating in the qualitative study were selected on a rational basis from the same database based on their high levels of explosive anger. The pattern of convergence between the data obtained from the quantitative and qualitative arms of the study adds support to the validity of our findings. The measures for psychological distress and anger were selected because these constellations of distress have been found to be common among women in previous studies in Timor-Leste. ${ }^{15}$ The K10 measure of psychological distress is widely used across cultures and has been found in previous studies to function well as a proxy measure for depression. ${ }^{23}$ The community measure for anger has been shown to converge with a gold standard structured clinical interview for IED. ${ }^{22}$ Together, these measures provide a general index of mental distress, noting that the K10 includes commonly experienced symptoms of depression, anxiety and somatisation. ${ }^{23}{ }^{24}$ The anger and psychological distress measures have been shown to correlate with disability and dysfunction. ${ }^{18} 29$

Limitations of the study are that it is cross-sectional in design, cautioning against drawing causal inferences regarding the relationships we found. It is possible that women with severe psychological distress are prone to focus excessively on the problem of bride price. Psychological distress may limit women's capacity to manage daily challenges which in Timor-Leste often concern economic hardships. Lia may represent one aspect of the duress caused by this broader economic stressor. In addition, the correlational nature of the data does not allow a conclusion to be drawn concerning directionality in judging the association between conflict with the spouse or family and bride price. Longitudinal studies are required to identify more precisely the sequencing of these factors, in particular, the extent to which resentment about bride price leads to psychological distress or vice versa. To some extent, however, our qualitative data address these issues by pointing to the complex interaction and compounding of stresses in generating distress among women. The descriptions women provided appeared to attest strongly to bride price playing an instrumental role in this outcome.

While we were able to establish an association between bride price and family and spousal conflict, we did not include a measure specific to assessing intimate partner violence. In addition, we did not explore the psychological or social impacts of bride price for men, although, anecdotally, our experience in Timor-Leste suggests that the practice is a source of substantial stress on that gender group. Future studies should address the impact of bride price on the mental health of husbands and their families.

Our findings are novel in pointing to a possible hidden source of mental distress among women in a postconflict country typified by Timor-Leste. Although the genesis of explosive anger among women is likely to be multifactorial (including exposure to male violence, past trauma and ongoing poverty caused by underdevelopment), the observation that bride price may 
contribute to this emotional response has important implications for the society. In particular, if the obligations imposed by bride price contribute to resentment towards the female partner by the husband and his family, then a vicious cycle may be set in train; poverty, resentment, heightened feelings of injustice, conflict with the spouse and family conflict may converge to intensify the women's emotional difficulties in a mutually reinforcing way, further exacerbating difficulties in the family. We have previously proposed a cycle of violence model for postconflict countries such as Timor-Leste in which past trauma exposure and patriarchal values contribute to violence against women, impacting adversely on the mother's mental well-being and the next generation. ${ }^{30}$ In that regard, past qualitative analysis of this dataset found strong associations between explosive forms of anger among women and a tendency to respond excessively when disciplining children. ${ }^{18}$ The present findings add a specific component to this model by identifying bride price as a possible contributor to the postulated cycle.

Whether bride price represented a similarly serious problem for women in pre-conflict times in Timor-Leste cannot be determined by our study. It seems likely, however, that bride price has led to greater difficulties in the contemporary epoch because of the increasing tension between traditional customs and the rapid social changes that have been ushered in by modernisation, accelerated in Timor-Leste by external intervention in the country following the period of mass conflict. Disruption of traditional family and community networks during the conflict may have undermined to some extent the sense of obligation that the younger generation feels to extended families in the payment of traditional gifts, contributions and offerings. Changing expectations and priorities, for example, in relation to the roles and status of women and the need to conserve funding for the education of children, may have lessened the motivation to pay bride price. The growth of the cash economy may have resulted in the inflation of bride price, creating conditions of long-term indebtedness in a setting of extreme poverty. Moreover, poverty associated with conflict has likely increased tension over resources and how they are spent. Other factors, not fully assessed in our study, such as changed expectations among women who have been educated, may add to resentment over bride price. For all these reasons, there may be a growing disjuncture between bride price as a functional custom designed to bond families together and the potential for that tradition to contribute to stress and family conflict.

In clinical settings, in Timor-Leste and other low income countries, all health professionals working with women should be aware of the possibility that bride price may be a factor contributing to mental distress and family conflict (including the potential for intimate partner violence). Any family intervention for women experiencing emotional distress in low-income and medium-income countries needs to consider the relevance of bride price, even if resolution of the problem may be difficult to achieve within a strictly clinical setting. More generally, at a society-wide level, any remedies proposed to overcome the stresses associated with bride price in postconflict countries such as Timor-Leste need to be carefully considered given the complexities of the cultural issues involved. From a policy perspective, leaders are faced with the difficult challenge of respecting and upholding traditions in the society while, at the same time, acknowledging that some customs, such as bride price, have undergone a transformation in their practice that can lead to psychological distress and family conflict in ways that are antithetical to promoting the status and equality of women. At the same time, if cultural change is to be achieved in a harmonious manner, it will need to be approached sensitively and incrementally. In that regard, the process of change is likely to be expedited more by the natural effects of modernisation and the adoption of new values than by specific measures instituted by policymakers. Engagement by leaders with the community, raising awareness of the possible unintended adverse consequences of bride price and encouraging alternative avenues for recognising traditional obligations may be a more viable approach than a more assertive attempt to curtail the practice, for example, by legislation.

\section{Handling editor Seye Abimbola}

Collaborators Alola Foundation, Timor-Leste.

Contributors SR conceived of the study, served as principal investigator throughout its execution and with DS and RT wrote the manuscript. SR, DS and RT received funding for the project. MM, AKT, DS, SR and SM managed and analysed the data. ES, SR and MF collected the data and conducted fieldwork. RT, SM, ES, MF, WT, SR, DS, MM and AKT were involved with contributing to revisions of the manuscript.

Funding Australian Research Council DP0987803 and National Health and Medical Research Council 568970.

Competing interests None declared.

\section{Patient consent Obtained.}

Ethics approval The University of New South Wales and the Ministry of Health, Timor-Leste.

Provenance and peer review Not commissioned; externally peer reviewed.

Data sharing statement Unpublished data have not been sought from outside parties; however, it may be available to them by negotiation.

Open Access This is an Open Access article distributed in accordance with the Creative Commons Attribution Non Commercial (CC BY-NC 4.0) license, which permits others to distribute, remix, adapt, build upon this work noncommercially, and license their derivative works on different terms, provided the original work is properly cited and the use is non-commercial. See: http:// creativecommons.org/licenses/by-nc/4.0/

\section{REFERENCES}

1. Gostin LO, Friedman EA. The sustainable development goals: one-health in the world's development agenda. JAMA 2015;314:2621-3.

2. Baingana F, Bannon I, Thomas R. Mental health and conflicts: conceptual framework and approaches. Washington DC: World Bank, 2005. 
3. Corno L, Voena A. Selling daughters: age of marriage, income shocks and bride price tradition. Rockwool Foundation Research Unit, 2015.

4. Ashraf N, Bau N, Nunn N, et al. Bride price and the returns to education for women. http://watson.brown.edu/files/watson/imce/ events/2015/Voena\%20Bride\%20Price\%20and\%20the\%20Returns\% 20to\%20Education\%20for\%20Women.pdf (accessed 21 July 2015).

5. Wendo C. African women denounce bride price. Lancet 2004;363:716.

6. Hague G, Thiara RK, Turner A. Bride-price and its links to domestic violence and poverty in Uganda: a participatory action research study. Womens Stud Int Forum 2011;34:550-61.

7. Posel D, Rudwick S. Marriage and Bridewealth (Ilobolo) in Contemporary Zulu Society. Afr Stud Rev 2014;57:51-72.

8. Kaye DK, Mirembe F, Johansson A, et al. Implications of bride price on domestic violence and reproductive health in Wakiso District, Uganda. Afr Health Sci 2007;5:300-3.

9. Niner S. Barlake: An exploration of marriage practices and issues of women's status in Timor-Leste. Local-Global: Identity, Security, Community 2012;11:138.

10. Khan N, Hyati S. Bride-price and domestic violence in Timor-Leste: a comparative study of married-in and married-out cultures in four districts id:164. Timor-Leste: UNFPA, 2012

11. Niner S. Effects and Affects: Women in the Post-conflict Moment in Timor-Leste: An Application of V. Spike Peterson's 'Gendering Insecurities, Informalization and War Economies'. The Palgrave Handbook of Gender and Development. Palgrave Macmillan UK, 2016:495-512.

12. Brandao CdE. Culture and its impact on social and community life: a case study of Timor-Leste. EWER Policy Brief. 2011;5. http://www cicr-columbia.org/wp-content/uploads/2011/02/Policy-Brief-5-Cultureand-its-Impact-on-Social-and-Community-Life.pdf

13. Cliggett L. Social components of migration: experiences from Southern Province, Zambia. Hum Organ 2000:59:125-35.

14. Corcoran-Nantes $Y$. The politics of culture and the culture of politics - a case study of gender and politics in Lospalos, Timor-Leste: analysis. Confl Secur Dev 2009;9:165-87.

15. Silove D, Liddell B, Rees $S$, et al. Effects of recurrent violence on post-traumatic stress disorder and severe distress in conflict-affected Timor-Leste: a 6-year longitudinal study. Lancet Global Health 2014;2:e293-300.

16. Abramsky T, Watts C, Garcia-Moreno C, et al. What factors are associated with recent intimate partner violence? Findings from the
WHO multi-country study on women's health and domestic violence. BMC Public Health 2011:11:109.

17. Devries $\mathrm{K}$, Watts $\mathrm{C}$, Yoshihama $\mathrm{M}$, et al. Violence against women is strongly associated with suicide attempts: evidence from the WHO multi-country study on women's health and domestic violence against women. Soc Sci Med 2011;73:79-86.

18. Rees S, Silove D, Verdial T, et al. Intermittent explosive disorder amongst women in conflict affected Timor-Leste: associations with human rights trauma, ongoing violence, poverty, and injustice. PLoS ONE 2013;8:e69207.

19. Dunn J. Genocide in East Timor. In: Totten S, Parsons W, eds. Century of genocide: critical essays and eyewitness accounts. New York: Routledge, 2009:265-95.

20. Creswell JW, Plano Clark VL, eds. Designing and Conducting Mixed Methods Research. Washington DC: SAGE, 2011.

21. Tong A, Sainsbury $P$, Craig J. Consolidated criteria for reporting qualitative research (COREQ): a 32-item checklist for interviews and focus groups. Int J Qual Health Care 2007;19:349-57.

22. Liddell B, Silove D, Tay K, et al. Achieving convergence between a community-based measure of explosive anger and a clinical interview for intermittent explosive disorder in Timor-Leste. J Affect Disord 2013;150:1242-6.

23. Patel V, Araya $\mathrm{R}$, Chowdhary $\mathrm{N}$, et al. Detecting common mental disorders in primary care in India: a comparison of five screening questionnaires. Psychol Med 2008;38:221-8.

24. Andrews $G$, Slade T. Interpreting scores on the Kessler Psychological Distress Scale (K10). Aust N Z J Public Health 2001;25:494-7.

25. Richards L. Data Alive! The Thinking Behind NVivo. Qual Health Res 1999;9:412-28.

26. Malterud K. Qualitative research: standards, challenges, and guidelines. Lancet 2001;358:483-8.

27. Tsai AC, Tomlinson M. Mental health spillovers and the Millennium Development Goals: the case of perinatal depression in Khayelitsha, South Africa. J Glob Health 2012;2:010302.

28. Brooks R, Silove D, Steel Z, et al. Explosive anger in postconflict Timor-Leste: interaction of socio-economic disadvantage and past human rights-related trauma. J Affect Disord 2011;131:268-76.

29. Silove D, Bateman C, Brooks R, et al. Estimating clinically relevant mental disorders in a rural and an urban setting in postconflict Timor-Leste. Arch Gen Psychiatry 2008;65:1205-12.

30. Rees S, Thorpe R, Tol W, et al. Testing a cycle of family violence model in conflict-affected, low-income countries: a qualitative study from Timor-Leste. Soc Sci Med 2015;130:284-91. 